\title{
La producción científica en el estudio de experiencia de usuario en educación: caso Web of Science y Scopus
}

\author{
Scientific production in the study of user \\ experience in education: case \\ Web of Science and Scopus
}

Cinthia De Oleo MORETA ${ }^{1}$ (D) 0000-0003-2173-6888

Elias SAID-HUNG ${ }^{2}$ (D) 0000-0002-0594-5906

\begin{abstract}
Resumen
El objetivo de este trabajo es realizar una aproximación sobre la evolución y rasgos generales de la producción científica divulgada en Web of Science y Scopus, relacionada con el estudio de la experiencia de usuario a nivel educativo. El trabajo analiza la totalidad de la producción registrada en los sistemas de indexación tomados como caso de estudio, 194 registros obtenidos a partir de los términos experiencia de usuario y educación. Se lleva a cabo un análisis de contenido de los resúmenes e información institucional, editorial y autores dispuestos en Web of Science y Scopus, además del análisis de los indicadores bibliométricos de cada trabajo identificado. Los datos obtenidos muestran que más de un $80 \%$ de la producción científica sobre el tema, mayoritariamente generada en eventos científicos, se ha producido en los últimos 5 años, bajo la autoría y afiliación institucional de entidades académicas y editoriales provenientes de los Estados Unidos. Esto daría cuenta de un marco académico nuevo, de creciente interés, alrededor del análisis de la experiencia de usuario desde una perspectiva educativa, condicionado por un debate altamente focalizado en una zona geográfica que está asumiendo un rol destacado en los planteamientos que están surgiendo sobre este tema a nivel científico.
\end{abstract}

Palabras clave: Educación. Experiencia de usuario. Producción científica. Usuarios. Scopus. Web of Science.

\begin{abstract}
This work has the goal to make an approximation of the evolution and general features present in scientific production disclosed in Web of Science and in Scopus, related to the study of user experience in education. The work analyzes the totality of the production registered in the indexation systems taken as a case of study, 194 records obtained from the use of terms user experience and edoucation. The study carries out a content analysis of the abstract and institutional, editorial and author information exposed in the Web of Science and Scopus, in addition to the analysis of the bibliometric indicators of each identified work. Obtained data show that more than $80 \%$ of the scientific production around the topic addressed, mostly generated in scientific events, has been produced in the last 5 years, under the

1 Universidad Internacional de La Rioja, Escuela Superior de Ingeniería y Tecnología. Máster en Diseño de Experiencia de Usuario, Av. de La Paz, 137, Logroño, 26006, La Rioja, España. Correspondencia para/Correspondence to: C. D. O. MORETA. E-mail: <cinthia.deoleo@unir.net>.

2 Universidad Internacional de La Rioja, Facultad de Educación, Máster en Educación Inclusiva e Intercultural. Logroño, La Rioja, España. Recibido el 13 del febrero del 2019, re-presentada el 16 del agosto del 2019 y aprobado el 25 del noviembre del 2019.
\end{abstract}

Como citar este artículo/How to cite this article

Moreta, C. D. O.; Hung, E. S. La producción científica en el estudio de experiencia de usuario en educación: caso Web of Sciencey Scopus. Transinformação, v. 32, e190003, 2020. http://dx.doi.org/10.1590/2318-0889202032e190003 
authorship and institutional affliation of academic institutions and publishers from the United States. This would point out to a new academic framework, of growing interest, around the analysis of the user experience from an educational perspective, conditioned by a highly focused debate on a geographical area, which is assuming a prominent role in the first approaches that are emerging on this subject, at the scientific level.

Keywords: Education. User experience. Scientific production. Users. Scopus. Web of Science.

\section{Introducción}

Hassan (2017) define la experiencia de usuario o "UX" - siguiendo el acrónimo que se le ha dado en inglés al momento de hablar de User Experience -, como una disciplina que tiene el objetivo de hacer que los productos tecnológicos sean amigables, fáciles de usar, y que permitan al usuario tener una experiencia satisfactoria con su uso de estos. A diferencia de la usabilidad, que hace referencia al diseño y desarrollo de productos o sistemas interactivos fáciles de utilizar, fáciles de aprender y de recordar cómo se utilizan, la UX añade la satisfacción al uso de un producto o servicio, pudiendo convertirse esta última en un elemento diferenciador y determinante para su éxito o fracaso. Por lo tanto, la usabilidad sería solamente un aspecto para considerar dentro de la UX.

Aunque la UX es un área de investigación reciente, desde que Norman (2010) utilizara por primera vez este término, profesionales e investigadores del área tecnológica, marketing y otras áreas de conocimiento han venido trabajando sobre esta temática. Un trabajo que se vio impactado por el cambio de perspectiva especializada que tenían los avances tecnológicos (mediados del siglo XX) y el paso a un período marcado por una masificación en el aprovechamiento de estos recursos por la sociedad (finales del siglo XX), en general, como respuesta al creciente interés que estaba adquiriendo el diseño del entorno, más allá de la simple funcionalidad de los equipos tecnológicos, en lo que se dio a conocer como Interacción Persona-Ordenador (IPO), conocida como Human-Computer Interaction (HCl) (Hewett et al., 1992). Un campo que, como bien apuntan Soler-Adillon et al. (2016) ha venido adquiriendo una larga tradición a nivel tecnológico, ya que ayudaría a entender los diferentes avances que se han dado en materia de diseño de interfaces, requeridos para el empleo de programas y dispositivo tecnológicos hasta la fecha.

El creciente interés en el estudio relacionado a la Interacción Persona-Ordenador trajo consigo la aplicación de métodos de investigación de otras disciplinas o áreas de conocimiento, tales como la ergonomía, la psicología y el diseño gráfico e industrial. Sin embargo, es sobre la década de 1990 cuando nace la usabilidad, al amparo de la $\mathrm{HCl}$, de la mano de autores como Nielsen (2012), considerado como uno de los referentes de esta nueva disciplina, al entender la usabilidad como un atributo que mide la calidad de las interfaces aplicadas a programas y dispositivos, de acuerdo con la facilidad de uso de estos. Algo que se enmarca en el nivel de cumplimiento de una serie de características, a saber: facilidad de aprendizaje, facilidad de memorización, eficiencia, pocos errores, y capacidad de generar satisfacción entre sus usuarios potenciales (Nielsen, 2012), lo que contribuyó al surgimiento de términos tales como diseño de interacción y arquitectura de información, ambos vinculados al diseño de programas y dispositivos tecnológicos y que aluden, en el primer caso, a la necesidad de prestar atención a la eficiencia práctica de las soluciones tecnológicas desde el punto de vista del usuario (Winograd, 1997) y, en el segundo caso, a la forma como se organiza y estructura la información en estos (Viñas, 2013). Conceptos que, como bien señalan autores como Albuquerque et al. (2011) y Hassan (2017), deben partir del conocimiento científico, creativo y artístico, combinando la rigurosidad, el talento y la experiencia con la funcionalidad y la estética visual, a nivel del diseño de interfaces, industrial y marketing, los cuales se engloban en lo que se entiende como UX.

Desde el punto de vista educativo, aunque se han publicado trabajos sobre UX afines a este campo de conocimiento, no se ha profundizado en estudios bibliométricos que muestren la evolución histórica del tema y que permitan tener una visión global de su proyección. El abordaje de la UX en la educación se ha enfocado en varias líneas de investigación, las cuales se han venido centrando en: 
(1) El empleo de la mecánica de juego, la gestión de proyectos y técnicas para la mejora de la experiencia de usuario en contextos académicos, como las bibliotecas académicas virtuales, entornos virtuales de aprendizaje y realidad virtual y aumentada (Kosmadoudi et al., 2013; Getto; Beecher, 2016; Koster et al., 2016; Sanchis-Font et al., 2017; Soler et al., 2017; Appleton, 2018; Zagorskis; Kapeniesks, 2018; Drosos et al., 2018; Fanfarelli et al. 2018; Arifin et al., 2018).

(2) El estudio y aplicación de métodos y modelos de UX para ser aplicados en educación, en lo que se refiere a la evaluación de sistemas de gestión de aprendizaje o Learning Management System (LMS) (Law et al., 2014; Hashim; Ahmad, 2015; Vorvoreanu; Connolly, 2015; Gray, 2016; Nakamura et al., 2017; Sahid et al., 2017).

(3) El estudio de procesos orientados a la adaptación de métodos de evaluación sin usuarios, como la evaluación heurística (método de evaluación desarrollado por Nielsen, 1994), para ser aplicado a la evaluación de software pedagógico (Gordon et al., 2016).

Cada una de estas líneas de investigación, no solo dan cuenta de algunas de las perspectivas que han sido objeto de análisis de la UX a nivel educativo, sino que sirven de referente del rápido crecimiento que ha venido adquiriendo este tema desde un punto de vista multidisciplinario, tanto a nivel académico como profesional, lo que justifica posiciones como la expuesta por Vorvoreanu et al. (2017) en lo que se refiere al carácter sustancial de generar estudios que ayuden a comprender el marco formativo que se está dando actualmente a su alrededor. Algo que exige también promover estudios como el expuesto en este trabajo, es decir, que ayuden a hacer una identificación inicial de los rasgos que han venido caracterizando la producción científica sobre este tema. Algo que ayudaría a entender qué, cómo y desde dónde se ha venido dando la aproximación y base conceptual que está condicionando la forma en que se está entendiendo, tanto el concepto de la UX, como la forma en que este se aplica desde un punto de vista educativo, por ejemplo.

\section{Procedimientos Metodológicos}

El objetivo de este trabajo es obtener una aproximación sobre la evolución y los rasgos generales presentes en la producción científica divulgada en Web of Science (2018) y en Scopus (2018), relacionada con el estudio de la UX a nivel educativo. De esta forma, ayudará a comprender mejor la frontera del conocimiento existente alrededor de la temática propuesta en la actualidad, al tener una mayor claridad con relación a las perspectivas conceptuales, metodológicas y enfoques temáticos abordados hasta la fecha.

El tratamiento del tema propuesto parte de la idea compartida en estudios realizados por autores como Hernández-González et al. (2016) o Granda-Orive et al. (2013), quienes apuntan a la utilidad que tienen las bases de datos bibliográficas como principales fuentes empleadas por estudios de carácter bibliométrico, como el realizado en este trabajo. Un propósito que dependerá siempre de la selección oportuna de bases de datos, en nuestro caso Web of Science y Scopus. Una escogencia hecha en vista de que, en el caso de la primera, además de ser una de las bases de datos más antiguas y de mayor impacto académico a nivel internacional, brinda herramientas de análisis que permiten valorar la calidad científica de las publicaciones que poseen. Mientras que en el caso de Scopus es esta la base de datos que cuenta con el mayor catálogo de resúmenes y citas de literatura científica (peer review) en la actualidad, con más de 21.000 títulos de revistas, 5.000 editores internacionales y 55 millones de registros, de acuerdo con lo expuesto por Pérez-Escoda (2017).

Lo indicado en el párrafo anterior justifica y da cuenta del porqué de la selección de estas dos bases de datos como espacios de búsqueda, recolección y análisis de los trabajos vinculados con la temática propuesta en este trabajo. Algo que también ha sido llevado a cabo por autores como Hernández-González et al. (2016) y Delgado, López-Cózar y Repiso (2013). Sobre todo, si lo que se quiere es avanzar en el desarrollo de trabajos de corte bibliométrico que ayuden a evaluar a la ciencia a través del uso complementario de la información dispuesta 
en estas dos principales bases de datos. No solo desde la comparación de factores de impacto o el índice h de los autores de los trabajos indexados, sino también haciendo uso de la información contenida en este tipo de escenarios bibliométricos para el análisis de la procedencia geográfica, idioma de edición y la editorial, entre otros aspectos generales, alrededor de la producción científica relativa a un determinado campo del saber. A la fecha no se tiene registro de que se hayan hecho estudios bibliométricos de este tipo alrededor del tema propuesto en este artículo (UX en la educación). Sobre todo, si se tiene en consideración que, en general, es un campo con menos de 10 años de recorrido cientííco, pero con un horizonte cada vez más importante en diferentes campos del saber, entre los que destaca la educación, como resultado del impacto que está trayendo consigo el uso de las Tecnologías de la Información y la Comunicación (TIC) en los procesos de enseñanza-aprendizaje.

Para alcanzar este objetivo, el trabajo parte de una investigación de tipo cualitativa, en la que se llevó a cabo un análisis de contenido de los resúmenes e información institucional, editorial y autores dispuestos en Web of Science y Scopus. Además, se realizó un análisis de los indicadores bibliométricos de cada trabajo identificado, con el fin de: (1) Identificar los tipos de producción académica registrados en los principales sistemas de indexación científica dispuestos en la actualidad. (2) Conocer la evolución que ha tenido el estudio del tema propuesto, desde un punto de vista histórico y científico. (3) Determinar las áreas geográficas que más influencia han tenido alrededor del estudio de la UX en la educación. (4) Establecer los rasgos institucionales y editoriales alrededor de los trabajos relacionados con el tema propuesto.

El estudio identificó, revisó, registró y analizó los trabajos registrados en la colección principal de Web of Science, formada por diez bases de datos (SCI-Expanded, SSCl, A\&HCl, ESCI, CPCI-S, CPCI-SSH, BKCl-S, BKCl-SSH, CCR-Expanded, IC), disponible en el portal Web of Knowledge. Mientras que en el caso de Scopus, se accedieron a los trabajos indexados en ella a través del portal SCImago. Como criterio de búsqueda de los trabajos analizados en este artículo, se utilizaron los términos UX y education, los cuales fueron combinados haciendo uso de la expresión boleana AND como principales palabras clave a la hora de seleccionar el conjunto de la muestra tomada en cuenta para alcanzar el objetivo general planteado.

La elección de las palabras clave tomadas para el desarrollo de este trabajo (UX AND education) se hizo partiendo del directo interés por identificar únicamente la producción científica cuyo abordaje se refiere al tema de la experiencia de usuario desde un punto de vista educativo, tanto en su título, como en el resumen o en las diferentes palabras clave registradas en los sistemas de indexación seleccionados en este estudio. Se tomó la expresión "UX y education" en inglés teniendo en cuenta que: (1) Toda la producción científica indexada en Web of Science y Scopus, más allá de que tengan un idioma principal ajeno al inglés, se indexan a nivel bibliométrico tomando el inglés como idioma de referencia. Por lo que el uso de los términos en inglés sirvió para identificar y consolidar toda la producción científica relacionada al tema aquí abordado. (2) Más allá de que nuestro interés se centra en tener una aproximación sobre cómo está siendo pensada y debatida la experiencia de usuario a nivel educativo, el uso del término UX, desde sus orígenes (Norman, 2010), ha sido al que se ha hecho mención generalizada dentro de las áreas donde se originó (tecnología y marketing, principalmente).

La búsqueda se realizó sin restricción temporal, con el fin de hacer el análisis histórico sobre el tema propuesto hasta la fecha de realización del trabajo de campo, llevado a cabo hasta el 10 de noviembre de 2018. Durante este proceso, se identificaron 194 trabajos científicos (53 trabajos registrados en la colección principal de Web of Science, y 141 trabajos registrados en Scopus) ${ }^{3}$, de los cuales 163 fueron analizados, teniendo en cuenta que en 31 de ellos (equivalente al 16\% del total identificado) se encontraban ambos sistemas de indexación.

Una vez identificada la totalidad de trabajos tomados como casos de estudio, se procedió a la revisión manual de títulos, resúmenes y palabras clave de cada uno. Además, se estableció también el enfoque teórico, la

\footnotetext{
3 Uno de los trabajos analizados, correspondiente a una publicación en un evento académico/científico, se encontraba en prensa cuando se llevó a cabo la extracción y análisis de la información.
} 
metodología utilizada, el alcance y ámbito de acción de dichos trabajos; así como los indicadores bibliométricos relacionados con el número de autores, la información institucional relacionada con los autores, el idioma, la editorial, el año de publicación, las fuentes de financiamiento y el número de citas en cada trabajo analizado.

Para el abordaje del tema propuesto, se emplearon las siguientes herramientas y/o programas: (1) ISI. exe (Leydesdorff, 2017) y MS Excel (Microsoft, Redmont, 2018), utilizados para identificar las fuentes científicas, los trabajos y los autores citados, la producción por instituciones, el análisis sobre la evolución de la producción científica, entre otros aspectos analizados. (2) VosViewer (Van Eck; Waltman, 2019) y Gephi (2017), empleados para construir y visualizar las redes bibliométricas de coocurrencia de palabras clave, palabras en los títulos y resúmenes. (3) GPS Visualizer (Portland, 2018), usado para elaborar los mapas de ubicación geográfica de las instituciones de los autores y de las editoriales. (4) WordArt (Microsoft, Redmont, 2018), (https://wordart.com), utilizado para generar las nubes de palabras clave que aparecen en el apartado de resultados.

\section{Resultados}

En primer lugar, hay que indicar que, de los 163 trabajos analizados, el 97,5\% de ellos se encontraban escritos en inglés y el resto (2,5\%) en otros idiomas (francés, alemán, español e italiano). Lo que lleva a observar una centralidad del tema abordado bajo un claro predominio de angloparlantes, restringiéndose el debate alrededor de la UX en educación a la posibilidad de comprensión o no de este idioma por parte de académicos y profesionales interesados en él.

El registro de trabajos extraídos en Web of Sciencey Scopus permite ver cómo más del 80,0\% del total de estos se ha producido en los últimos 5 años, siendo el primer caso de estudio de 2003. Esto muestra cómo, además de ser un tema novedoso con poco más de 15 años de registros de trabajos científicos en estos sistemas de indexación, en los últimos años es cuando se ha producido un verdadero auge de la producción científica interesada en abordar el tema de la UX a nivel educativo.

El tipo de producción registrado en Web of Science y Scopus se ha concentrado mayormente en artículos de eventos académicos y/o científicos (65,5\% de los trabajos analizados), mientras que el restante porcentaje está compuesto por artículos publicados en revistas cientíicas (28,9\%), libros y capítulos de divulgación científica $(3,04 \%)$ y editoriales $(0,50 \%)$. Una producción en la que se observó una media de 2,4 autores y donde la mayor parte de estos pertenecen a instituciones de Estados Unidos (Tabla 1), lo que reafirmaría la concentración que se estaría dando a nivel de conocimiento, alrededor del tema propuesto, desde el punto de vista institucional y geográfico.

Tabla 1. Top 10 de los autores e instituciones con trabajos publicados en Web of Sciencey Scopus.

\begin{tabular}{lll}
\hline Autores & Instituciones de los autores & Trabajos \\
\hline Getto, G. & East Carolina University (United States) & 3 \\
Gray, C. M. & Purdue University (United States) & 3 \\
Vorvoreanu, M. & Purdue University (United States) & 3 \\
Seyam, M. & Virginia Tech (United States) & 3 \\
Fleming-May, R.; Mays, R.; Walker, T.; Forrester, A.; Tenopir, C.; Bilal, D.; Allard, S. & University of Tennessee (United States) & 2 \\
Donohue, S. K.; Richards, L. G. & University of Virginia (United States) & 2 \\
McCrigler, B. & IFixit (United States) \\
Elliott, L. J. & Missouri Western State University (United States) \\
Yang, Q. & Carnegie Mellon University (United States) & 2 \\
Spartz, J. M.; Weber, R. P. & University of Wisconsin (United States) & 2 \\
\hline
\end{tabular}

Fuente: Elaboración propia (2018). 
Pese el contexto colaborativo presente en la generación de conocimiento alrededor del tema propuesto (ante la media de autores identificados en el conjunto de trabajos analizados), los datos recolectados y analizados en este trabajo muestran un escenario que debería ser tenido en consideración al momento de estimar el potencial impacto que pudiese traer consigo el hecho de que el tema de estudio está siendo abordado por autores, instituciones y escenarios académicos-científicos concretos, en la mayoría de los casos con un factor de impacto medio-bajo o bajo (Tabla 2). Sobre todo, si se tiene en cuenta que la mayoría de estos autores y espacios de debate científico alrededor del tema abordado en este artículo se han venido ubicando a nivel editorial en los Estados Unidos y en países europeos, lo que relegaría al resto de regiones del mundo a un segundo plano (e.g. América Latina, Asia o Europa del Este), a la hora de establecer las bases conceptuales y aproximaciones prácticas que se han venido haciendo hasta ahora alrededor de la UX a nivel educativo. Esto condiciona, además, los marcos de referencia e intereses que pudiesen darse alrededor de la UX a nivel educativo a temas, enfoques metodológicos y teóricos afines a los promovidos en los países y regiones antes mencionados.

En cuanto a los resultados del análisis de títulos, resúmenes y palabras clave, cabe destacar que los trabajos analizados mayormente se centran en el diseño y evaluación de aplicaciones o herramientas y el estudio y adaptación de metodologías y modelos basados en la experiencia de usuario, aplicados a la gamificación, juegos serios, e-learning, realidad virtual, aprendizaje automático, gestión de proyectos, sistemas de gestión de aprendizaje, bibliotecas virtuales y simulación (Figura 1). Y, en términos generales, se trata de trabajos que, desde el punto

Tabla 2. Top10 de eventos y revistas indexadas en Web of Science y Scopus, en el área de UX en Educación.

\begin{tabular}{|c|c|c|c|c|c|}
\hline \multicolumn{6}{|c|}{ Eventos } \\
\hline \multicolumn{3}{|l|}{ Nombre del evento } & País & Docs & $\%$ \\
\hline \multicolumn{3}{|l|}{ Lecture Notes in Computer Science } & United States & 23 & 11,86 \\
\hline \multicolumn{3}{|l|}{ ACM International Conference Proceeding Series } & United States & 6 & 3,09 \\
\hline \multicolumn{3}{|c|}{ Conference on Human Factors in Computing Systems - Proceedings } & United States & 4 & 2,06 \\
\hline \multicolumn{3}{|l|}{ IEEE International Professional Communication Conference } & United States & 4 & 2,06 \\
\hline \multicolumn{3}{|l|}{ Communications in Computer and Information Science } & Switzerland & 3 & 1,55 \\
\hline \multicolumn{3}{|c|}{ ACM SIGCHI Conference on Human Factors in Computing Systems (CHI) } & United States & 2 & 1,03 \\
\hline \multicolumn{3}{|c|}{ Proceedings of the 2015 International Conference on Collaboration Technologies and Systems } & United States & 2 & 1,03 \\
\hline \multicolumn{3}{|c|}{ International Journal of Sociotechnology and Knowledge Development } & Switzerland & 2 & 1,03 \\
\hline \multicolumn{3}{|c|}{ DIS 2018 - Proceedings of the 2018 Designing Interactive Systems Conference } & United States & 2 & 1,03 \\
\hline \multicolumn{3}{|l|}{ CEUR Workshop Proceedings } & Canada & 2 & 1,03 \\
\hline \multicolumn{6}{|c|}{ Revistas } \\
\hline Nombre de la Revista & Cuartil & Editorial & País & Docs & $\%$ \\
\hline Advances in Intelligent Systems and Computing & Q4 & Springer & Switzerland & 3 & 1,55 \\
\hline Cartographic Perspectives & Q3 & NACIS & United States & 2 & 1,03 \\
\hline $\begin{array}{l}\text { Communications in Computer and Information } \\
\text { Science }\end{array}$ & Q3 & Springer & Switzerland & 3 & 1,03 \\
\hline Fujitsu Scientific and Technical Journal* & Q4 & Fujitsu Ltd & Japan & 2 & 1,03 \\
\hline $\begin{array}{l}\text { International Journal of Sociotechnology and } \\
\text { Knowledge Development }\end{array}$ & Q3 & |Gl Global & United States & 2 & 1,03 \\
\hline Procedia Computer Science ${ }^{* *}$ & & Elsevier & Netherlands & 2 & 1,03 \\
\hline Performance Measurement and Metrics & Q1 & Emerald Group Publishing Ltd & England & 2 & 1,03 \\
\hline
\end{tabular}

Fuente: Elaboración propia (2018).

Nota: ( ${ }^{*}$ Única revista de la tabla indexada en Web of Science, por lo que el cuartil indicado en las demás revistas no corresponde a Web of Science, sino a Scopus. $\left({ }^{* *}\right)$ Revista indexada en Scopus, pero aún no tiene asignado un cuartil. 
de vista metodológico, se centran en estudios experimentales o cuasiexperimentales (45,2\%), estudios de casos $(19,8 \%)$, trabajos descriptivos con enfoque cualitativo (15,2\%), trabajos descriptivos con enfoque mixto (5,0\%), estudios reflexivos y comparativos (12,2\%) y, en menor proporción, exploratorios (3,0\%).

Otro aspecto destacable es que el 67,0\% de los trabajos analizados centran el estudio de la UX en contextos de educación universitaria, mientras que el resto (33,0\%), a ámbitos de educación primaria y secundaria (13,2\%), profesional (8,0\%) y al abordaje del tema desde una perspectiva general $(12,0 \%)$. Además, para el 74,0\% de los trabajos analizados se había delimitado el alcance a nivel nacional, es decir, apenas 3 de cada 10 trabajos analizados abordaron el tema objeto de este análisis desde un contexto internacional y comparado.

El análisis de las palabras clave vinculadas a la producción científica analizada (Figura 1 y Figura 2) permite reconocer dos etapas o períodos en los enfoques de los estudios abordados alrededor del tema estudiado en este trabajo: (a) La primera etapa, que abarca desde los estudios iniciales en el año 2003 hasta el año 2014. Esta etapa comprende mayormente estudios generales de tipo descriptivo sobre UX, aplicado a la gamificación, e-learning, diseño de interfaces, y más concretamente, al diseño de interacción. (b) La segunda etapa, (que concentra el 70,0\% de la producción científica analizada en este trabajo) comprende desde 2015 hasta 2018. A pesar de mantenerse la presencia de conceptos como UX, e-learning, diseño o gamificación, se aprecia un cambio de tendencia a la hora de establecer aproximaciones científicas alrededor de esta temática, las cuales comienzan a ser cada vez más específicas (e.g. la aplicación práctica de técnicas de diseño centrado en el usuario, el diseño y evaluación de aplicaciones o herramientas, modelos basados en la experiencia de usuario y plataformas de sistemas de gestión de aprendizaje o bibliotecas virtuales).

En resumen, la primera etapa comprende estudios teóricos más generales, mientras que la segunda etapa aborda un conocimiento de corte más práctico, orientado a la aplicación y prueba de conceptos, métodos y técnicas que dan lugar a un nivel de especialización cada vez mayor.

La media de citas recibidas en los 163 trabajos analizados es de 7. Los casos más referenciados, mostrados en la Tabla 3 a modo de ejemplo, están relacionados con el uso de estrategias para la mejora de la experiencia de
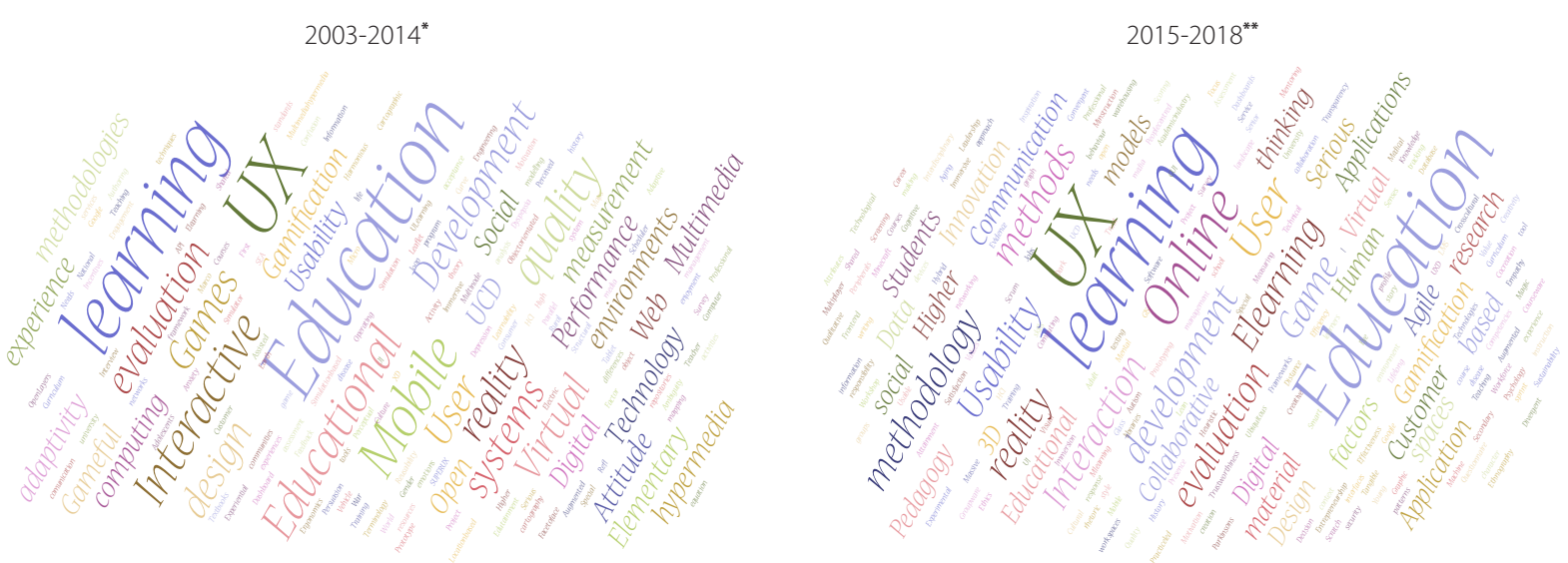

Figura 1. Nube de palabras a partir de palabras clave en producción científica indexada en Web of Science y Scopus.

Nota: *Del 2003 al 2014 el análisis comprende 44 casos. **Del 2015 al 2018, comprende 119 casos. La nube de palabras se ha creado con WordArt. La normalización de los términos es realizada por la herramienta de forma automática. La herramienta analiza todas las palabras utilizadas, y da mayor importancia a los términos que aparecen más veces, de modo que dichos términos aparecen con mayor tamaño. Los términos compuestos los analiza como términos separados, y elimina de forma automática el uso del guion (-).

Fuente: Elaboración propia (2018). 


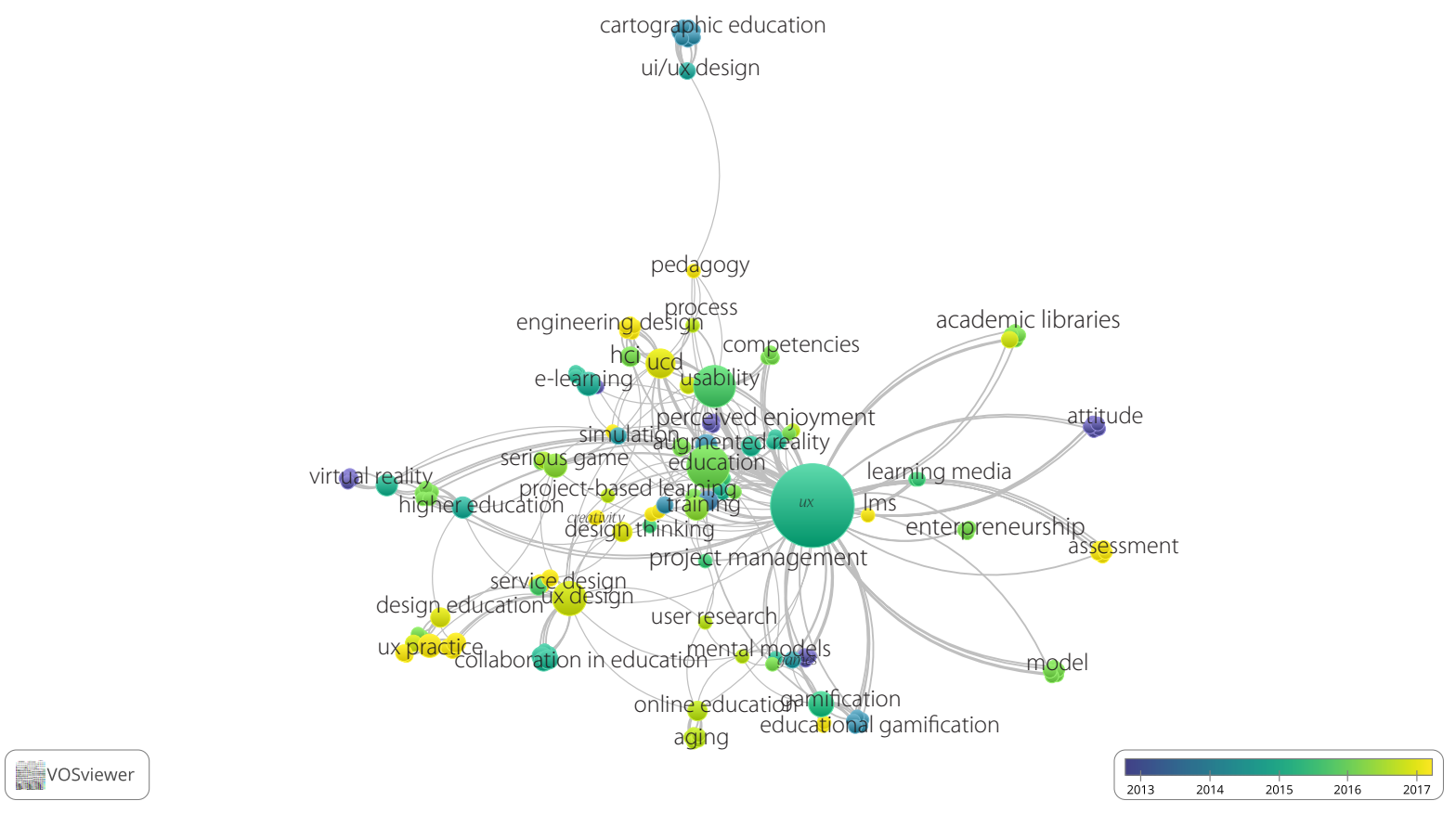

Figura 2. Mapa de palabras clave vinculadas a la producción científica indexada en Web of Sciencey Scopus.

Nota: Se muestran solamente los términos que han aparecido un mínimo de 5 ocasiones en los títulos y resúmenes analizados. El gráfico se ha realizado con el programa VosViewer, que agrupa por colores los clústers de palabras y asigna el tamaño a los nodos según su presencia en la red. Fuente: Elaboración propia (2018).

usuario con elementos del juego, diseño de UX y modelos, técnicas y métricas de UX, lo que daría cuenta de un contexto de interés que marcaría la producción de un nuevo conocimiento científico, alrededor de los trabajos registrados y analizados en Web of Science y Scopus. Un conocimiento que, al menos hasta la fecha de desarrollo de este estudio, se estaría centrando en áreas de conocimiento más vinculadas a la ingeniería, las ciencias de la computación y la información que a la educación y/o ciencias sociales. Lo que resultaría lógico teniendo en cuenta de dónde parte el término UX (ingeniería y ciencias de la computación), aunque también ayuda a ver cómo sigue estando ausente el abordaje de este tema desde una perspectiva más cercana a las ciencias sociales y la educación, lo que condicionaría la aproximación que se estaría dando en la actualidad a este tema, al predominar la óptica técnica de la mejora de la experiencia del usuario sobre otras perspectivas que aborden este tema desde el potencial impacto pedagógico y social que pudiese traer consigo ello, por ejemplo.

\section{Discusión y Conclusión}

Uno de los aspectos más destacables de los resultados obtenidos es que la mayoría de los trabajos analizados corresponden a comunicaciones de congreso, siendo éste el escenario que ha dado mayor visibilidad al tema analizado hasta la fecha, lo que daría cuenta, como se hizo mención en el apartado anterior, de un contexto científico novedoso o de creciente interés alrededor del tema estudiado. Pero, sobre todo, muestra que en este momento la evolución histórica del debate y desarrollo científico alrededor del tema estudiado pareciera haber pasado de los estudios descriptivos generales a estudios de corte más prácticos y de aplicación de conceptos y métodos, los cuales, por ejemplo, han sido publicados mayoritariamente en revistas con un factor de impacto 
Tabla 3. Top 10 de los trabajos y autores más referenciados en producción cientíica indexada en Web of Science y Scopus sobre de UX en Educación.

\begin{tabular}{|c|c|c|c|}
\hline \multicolumn{4}{|c|}{ Trabajos analizados con más citas } \\
\hline Autor(es) & $\begin{array}{l}\text { Tipo de producción } \\
\text { científica }\end{array}$ & Título & Citas \\
\hline Kosmadoudi, Z.; Lim, T.; Ritchie, J.; Louchart, S.; Liu, Y.; Sung, R. & $\mathrm{C}$ & $\begin{array}{l}\text { Engineering design using game-enhanced } \\
\text { CAD: The potential to augment the user } \\
\text { experience with game elements }\end{array}$ & 230 \\
\hline Law, E. L. C.; Van Schaik, P.; Roto, V. & C & $\begin{array}{l}\text { Attitudes towards user experience (UX) } \\
\text { measurement }\end{array}$ & 110 \\
\hline Rubio, G.; Navarro, E.; Montero, F. & C & $\begin{array}{l}\text { APADYT: a multimedia application for SEN } \\
\text { learners }\end{array}$ & 76 \\
\hline Dove, G.; Halskov, K.; Forlizzi, J.; Zimmerman, J. & C & $\begin{array}{l}\text { UX Design Innovation: Challenges for Working } \\
\text { with Machine Learning as a Design Material }\end{array}$ & 74 \\
\hline Roth, R. E. & A & $\begin{array}{l}\text { Rethinking cartography curriculum to train the } \\
\text { contemporary cartographer }\end{array}$ & 62 \\
\hline Gray, C.M. & A & $\begin{array}{l}\text { It's More of a Mindset Than a Method: UX } \\
\text { Practitioners' Conception of Design Methods }\end{array}$ & 60 \\
\hline $\begin{array}{l}\text { Fleming-May, R.; Mays, R.; Walker, T.; Forrester, A.; Tenopir, C.; } \\
\text { Bilal, D.; Allard, S. }\end{array}$ & C & $\begin{array}{l}\text { Experience assessment: designing an innovative } \\
\text { curriculum for assessment and UX professionals }\end{array}$ & 49 \\
\hline Getto, G.; Beecher, F. & C & $\begin{array}{l}\text { Toward a Model of UX Education: Training UX } \\
\text { Designers Within the Academy }\end{array}$ & 49 \\
\hline Appleton, L. & C & $\begin{array}{l}\text { Qualitative methods for engaging students in } \\
\text { performance measurement }\end{array}$ & 49 \\
\hline Conradie, P. D.; Vandevelde, C.; De Ville, J.; Saldien, J. & A & $\begin{array}{l}\text { Prototyping Tangible User Interfaces: Case } \\
\text { Study of the Collaboration between Academia } \\
\text { and Industry }\end{array}$ & 46 \\
\hline \multicolumn{4}{|c|}{ Autores referenciados en producción científica analizada e indexada en Web of Science y Scopus } \\
\hline Autor(es) & & Área de conocimiento & Citas \\
\hline Kosmadoudi, Z.; Lim, T; Ritchie, J.; Louchart, S.; Liu, Y.; Sung, R. & & $\begin{array}{l}\text { Ciencias de la Computación, Ingeniería de } \\
\text { Software }\end{array}$ & 230 \\
\hline Law, E. L. C.; Van Schaik, P.; Roto, V. & & Ciencias de la Computación, Ingeniería, Psicología & 110 \\
\hline Rubio, G.; Navarro, E.; Montero, F. & & $\begin{array}{l}\text { Ciencias de la Computación, Ingeniería, } \\
\text { Psicología }\end{array}$ & 78 \\
\hline Dove, G.; Halskov, K.; Forlizzi, J.; Zimmerman, J. & & Ciencias de la Computación & 74 \\
\hline Roth, R. E. & & Geografía & 74 \\
\hline Balog, A.; Pribeanu, C. & & Automática y Sistemas de Control & 62 \\
\hline Gray, C. M. & & Ciencias de la Computación & 62 \\
\hline Appleton, L. & & Ciencias de la Información & 49 \\
\hline Fleming-May, R.; Mays, R.; Walker, T.; Forrester, A.; Tenopir, C.; Bil & al, D.; Allard, S. & Ciencias de la Información & 49 \\
\hline Conradie, P. D.; Vandevelde, C.; De Ville, J.; Saldien, J. & & Educación, Ingeniería & 46 \\
\hline
\end{tabular}

Nota: De estos 26 trabajos más citados correspondientes a Comunicaciones de Congreso, 23 corresponden a Web of Science, mientras que 3 corresponden a Scopus, y 3 trabajos se encuentran indexados en ambas bases de datos. Los 10 artículos más citados corresponden a Wos, mientras que solamente 2 de ellos se encuentran indexados en ambas bases de datos.

Fuente: Elaboración propia (2018)

medio-bajo y bajo, en los últimos 5 años. Algo que pareciera lógico que avance en unos años a la visibilidad del tema en escenarios académicos con un mayor factor de impacto y/o relevancia, pero sobre todo a la aplicación de estudios de corte comparativo entre casos y/o países. Lo que ayudará a dar mayor profundidad al estudio de la UX a nivel educativo. 
El número de casos analizados en este trabajo, extraídos de los principales sistemas de indexación académica actual a nivel internacional (Web of Sciencey Scopus), es un dato interesante que amerita también ser destacado. El número acotado de trabajos identificados mostraría un contexto no necesariamente de baja producción científica, sino de la baja divulgación que aún tiene el tema abordado en ámbitos de impacto y relevancia científica como los tenidos en cuenta para la realización de este estudio. Algo que no solo mostraría la influencia que pudiesen tener también otros ámbitos ajenos a la academia alrededor del tema UX como, por ejemplo, las publicaciones y escenarios de debate profesional y/o de impacto académico eminentemente especializado (e.g. Philosopher Index, en el campo humanístico), nacionales (e.g. Publindex, en Colombia) o regionales (e.g. Scielo o Latindex, a nivel de América Latina), sino también el punto en el que se encuentra, donde en los últimos 5 años se concentra el 80\% de los trabajos analizados y que sería interesante promover nuevos estudios que permitan hacer un seguimiento continuado del tema aquí abordado, con el fin de ayudar a identificar nuevas tendencias y rasgos de la producción científica de éste.

Los resultados mostrados también marcan rasgos etnocéntricos en el estudio de la UX a nivel educativo, al observarse un claro predominio de autores, instituciones y editoriales estadounidenses y europeos. Esto sin dejar de lado el dominio que tienen aún las áreas de conocimiento afines a las ingenierías y ciencias de la computación, en contra de otras áreas de conocimiento que han tenido menor representación hasta ahora (las ciencias sociales y la educación). Algo que marcaría, no solo, el interés que ha venido teniendo el estudio de la UX a nivel educativo desde contextos institucionales, geográficos y áreas de conocimientos concretas, sino del potencial nivel de condicionamiento que ello pudiese traer consigo en los potenciales abordajes del tema estudiado, de acuerdo con los intereses y perspectivas dominantes de unos actores científicos altamente focalizados que no necesariamente representan la diversidad de realidades que pudiesen ser atendidos a la hora de medir el potencial impacto alrededor del tema estudiado, por ejemplo en contextos de países considerados emergentes (e.g. países asiáticos) o en vías de desarrollo (e.g. países latinoamericanos).

Finalmente, más allá de las limitaciones inherentes del estudio aquí mostrado, consideramos que el abordaje de un tema como el propuesto, aunque se haya hecho a nivel descriptivo, aporta elementos importantes. Sobre todo, al momento de estimar cómo está siendo el abordaje de la UX desde el punto de vista educativo, a nivel académico. Algo que se ha realizado, desde la producción científica publicada hasta la fecha de realización de este estudio, en revistas adscritas a dos de los principales sistemas de indexación científica en la actualidad (Web of Science y Scopus), lo que ayuda a evaluar y estimar el estado de avance de la ciencia que rodea el proceso aquí abordado, cada vez más relevante a la hora de avanzar en procesos de aplicación de las TIC para favorecer procesos de enseñanza-aprendizaje, tanto desde escenarios educativos formales como informales. Una realidad que, al menos según los datos recabados y analizados previamente, nos permite ver que el debate alrededor del eje UX y la educación pareciera no estar dándose aún desde escenarios científicos como los analizados, sino en contextos ajenos. Y que el poco conocimiento científico divulgado indexado a la fecha se encuentra centralizado a nivel de editoriales anglosajonas, ajenas a contextos formalmente educativos. Lo que representa una limitación desde el punto de vista didáctico y/o pedagógico, por ejemplo, de la centralidad del debate que se ha hecho hasta la fecha, más atento a cuestiones técnicas o prácticas, desde el punto de vista de la ingeniería, el diseño digital y las ciencias de la computación. Por lo que, consideramos que resultaría importante avanzar en la promoción de nuevos trabajos bibliométricos o de otro tipo de enfoque metodológico, que tengan interés en analizar comparativamente los resultados aquí expuestos con sistemas de indexación académicos y/o científicos tales como, por ejemplo, Scielo y Latindex, lo que seguramente ayudaría a profundizar en los puntos destacados en este apartado, así como reconocer otros contextos geográficos y editoriales que pudiesen estar siendo sensibles en el abordaje del tema aquí planteado. 
C. D. O. MORETA contribuido a extracción, análisis e interpretación de los datos, y elaboración del manuscrito. E. SAID-HUNG el concepto, diseño, y revisión de la versión final del artículo.

\section{Referencias}

Albuquerque, A.; Rodriguez R.; Marques, L. Sobre os fundamentos da arquitetura da informação. Perspectivas em Gestão e Conhecimento, v. 1, p. 60-72, 2011. Número especial.

Appleton, L. Qualitative methods for engaging students in performance measurement. Information and Learning Science, v. 119, n. 1-2, p. 64-76, 2018.

Arifin, Y.; Sastria, T. G.; Barlian, E. User experience metric for augmented reality application: a review. Procedia Computer Science, v. 135, p. 648-656, 2018.

Drosos, V. et al. A 3D serious game for cultural education. In: International Conference on Information, Intelligence, Systems and Applications, 8, 2017, Lanarca. Proceedings [...]. Lanarca: IEEE Xplore, p. 1-5, 2018.

Fanfarelli, J. R.; McDaniel, R.; Crossley, C. Adapting UX to the design of healthcare games and applications. Entertainment Computing, v. 28, p. 21-31, 2018.

Gephi for Windows. San Francisco: Gephi. 2017. Disponible en: https://gephi.org/users/download/. Acceso en: 12 nov. 2018.

Getto, G.; Beecher, F. Toward a model of UX education: training UX designers within the academy. IEEE Transactions on Professional Communication, v. 59, n. 2, p. 153-164, 2016.

Gordon, N.; Brayshaw, M.; Aljaber, T. Heuristic evaluation for serious immersive games and $\mathrm{M}$-instruction. Lecture Notes in Computer Science, v. 9753, p. 310-319, 2016.

GPS Visualizer. GPS Visualizer: Do-It-Yourself mapping. Portland: GPS Visualizer, 2018. Available from: https://www. gpsvisualizer.com/. Cited: Nov. 122018.

Gray, C. M. It's more of a mindset than a method: UX practitioners' conception of design methods. In: Conference on Human Factors in Computing Systems, 2016, San Jose, California. Proceedings [...]. New York: ACM, p. 4044-4055, 2016.

Granda-Orive, J. I. et al. Ciertas ventajas de Scopus sobre Web of Science en un análisis bibliométrico sobre tabaquismo. Revista Española de Documentación Científica, v. 36, n. 2, p. 1-9, 2013.

Hashim, A. S.; Ahmad, W. F. W. A study on usability of mobileschool system for secondary school: role-based questionnaire method. Lecture Notes in Computer Science, v. 9429, p. 393-404, 2015.

Hassan, Y. Experiencia de usuario: principios y métodos. [S.I: s.n.], 2017.

Hernández-González, V. et al. Comparación entre Web of Science y Scopus, estudio bibliométrico de las revistas de anatomía y morfología. International Journal of Morphology, v. 34, n. 4, p. 1369-1377, 2016.
Hewett, T. et al. Human-Computer interaction. In: Hewett, T. et al. ACM SIGCHI Curricula for Human-Computer Interaction. New York: ACM, 1992. p. 5-27. Available from: http://old.sigchi. org/cdg/cdg2.html\#2_1. Cited: Dec. 21, 2018.

Kosmadoudi, Z. et al. Engineering design using game-enhanced CAD: the potential to augment the user experience with game elements. CAD Computer Aided Design, v. 45, n. 3, p. 777-795, 2013.

Koster, A. et al. Towards a digital teaching platform in brazil: findings from UX experiments. Lecture in Notes in Computer Sciences, v. 9753, p. 685-694, 2016.

Law, E. L. C.; Van Schaik, P.; Roto, V. Attitudes towards user experience (UX) measurement. International Journal of Human Computer Studies, v. 72, n. 6, p. 526-541, 2014.

Leydesdorff, L. ISI.exe. Amsterdam: Loet Leydesdorff, 2017. Available from: https://www.leydesdorff.net/software/isi/index. html/. Access: Dec. 21, 2018.

López-Cózar, E. D.; Repiso, R. El impacto de las revistas de comunicación: comparando Google Scholar Metrics, Web of Science y Scopus. Comunicar, v. 21, n. 41, p. 45-52, 2013.

Microsoft. Power Point: word art. Redmont: Microsoft Corporation, 2018. Disponible en: https://products.office. com/pt-br/powerpoint. Acceso en: 1 nov. 2018.

Microsoft. MS Excel. Redmont: Microsoft Corporation, 2018. Disponible en: https://products.office.com/es-es/excel. Acceso en: 1 nov. 2018.

Nakamura, W. T.; Oliveira, E. H. T.; Conte T. Usability and user experience evaluation of learning management systems a systematic mapping study. In: International Conference on Enterprise Information Systems, 19, 2017, Porto, Portugal. Proceedings [...]. Porto: ICEIS, v. 3, p. 97-108, 2017.

Nielsen, J. Usability inspection methods. In: Conference on Human Factors in Computing Systems, 1994, Boston, Massachusetts. Proceedings [...]. New York: AMC, p. 413-414, 1994.

Nielsen, J. Usability 101: introduction to usability. Fremont: Nielsen Norman Group, 2012. Available from: https://www. nngroup.com/articles/usability-101-introduction-to-usability/. Access: Dec. 212018.

Norman, D. La psicología de los objetos cotidianos. San Sebastián: Nerea, 2010.

Pérez-Escoda, A. WoS y Scopus: los grandes aliados de todo investigador. Comunicar, 2017. Disponible en: https:// comunicarautores.com/2017/02/09/wos-y-scopus-los-grandesaliados-de-todo-investigador/. Acceso en: 21 jul. 2019.

Sahid, D. S. S. et al. Evaluation and measurement of learning management system based on user experience. In: International 
Annual Engineering Seminar, 6, 2016, Yogyakarta. Proceedings [...]. Yogykarta: IEEE Xplore, p. 72-77, 2017.

Sanchis-Font, R. et al. Improving the virtual learning experience: user-centered design in e-Learning. In: International Conference on Technology, Education and Development, 11, 2017, Valencia, Spain. Proceedings [...]. Valencia: Inted, p. 9902-9907, 2017.

Scopus. Alcobendas: Fundación Española de Ciencia y Tecnología, 2018. Disponible en: https://www.fecyt.es/es/ recurso/scopus. Acceso en: 11 nov. 2018.

Soler-Adillon, J. et al. Perfil del profesional de la comunicación interactiva: fundamentos, actualidad y perspectiva. El Profesional de la Información, v. 25, n. 2, 2016. Disponible en: https://recyt.fecyt.es/index.php/EPI/article/view/epi.2016. mar.06. Acceso en: 21 dec. 2018.

Soler, J. L. et al. The power of sight: using eye tracking to asses learning experience in virtual reality environments. In: International Conference on Technology, Education and Development, 11, Valencia, Spain, 2017. Proceedings [...]. Valencia: Inted, p. 8684-8689, 2017.

Van Eck, N. J.; Waltman, L. VOSviewer. Leiden: VOSviewer, 2019. Available from: www.vosviewer.com. Access: Dec. 212018.

Viñas, M. Arquitectura de sitios Web de bibliotecas universitarias: el sistema de bibliotecas de la Universidad
Nacional de La Plata, Argentina. Palabra clave, v. 3 n. 1, p. 52-64, 2013.

Vorvoreanu, M.; Connolly P. E. Using an experience design approach to curriculum creation. In: American Society for Engineering Education Annual Conference and Exposition, 22, 2015, Seattle. Proceedings [...]. Washington: American Society for Engineering Education, 2015.

Vorvoreanu, M. et al. Advancing UX education: a model for integrated studio pedagogy. In: Conference on Human Factors in Computing Systems, 2017, Denver, Colorado. Proceedings [...]. New York: ACM, p. 1441-1446, 2017.

Web of Science. Alcobendas: Sistema Español de Ciencia y Tecnología, 2018. Disponible en: https://www.fecyt.es/es/ recurso/web-science. Acceso en: 11 nov. 2018.

Winograd, T. From computing machinery to interaction design. In: Denning, P.; Metcalfe, R. Beyond calculation the next fifty years of computing. New York: Springer, 1997. p. 149-162. Available from: http://hci.stanford.edu/ winograd/papers/ acm97.html. Access: Dec. 212018.

Zagorskis, V.; Kapenieks, A. Impact of LMS selection on students' activity students' activity evaluation problems in moodle and open edX learning management systems. In: International Conference on Computer Supported Education, 10, 2018, Funchal, Madeira, Portugal. Proceedings [...]. Funchal: Csedu, v. 1, p. 505-512, 2018. 